\title{
Breast cancer subtypes and outcome after local and regional relapse
}

\author{
E. Montagna ${ }^{1 \star}$, V. Bagnardi ${ }^{2,3,4}$, N. Rotmensz ${ }^{2}$, G. Viale ${ }^{5,6}$, G. Renne ${ }^{5,6}$, G. Cancello ${ }^{1}$, \\ A. Balduzzi ${ }^{1}$, E. Scarano ${ }^{1}$, P. Veronesi ${ }^{5,7}$, A. Luini ${ }^{7}$, S. Zurrida ${ }^{5,7}$, S. Monti ${ }^{7}$, M. G. Mastropasqua ${ }^{6}$, \\ L. Bottiglieri ${ }^{6}$, A. Goldhirsch ${ }^{1} \&$ M. Colleoni ${ }^{1}$ \\ ${ }^{1}$ Department of Medicine; ${ }^{2}$ Division of Epidemiology and Biostatistics, European Institute of Oncology; ${ }^{3}$ Department of Statistics, University of Milan-Bicocca, Milan, Italy; \\ ${ }^{4}$ Frontier Science and Technology Research Foundation Southern Europe, Chiasso, Switzerland; ${ }^{5}$ University of Milan School of Medicine, Milan; ${ }^{6}$ Division of Pathology; \\ ${ }^{7}$ Division of Senology, European Institute of Oncology, Milan, Italy
}

Received 20 January 2011; revised 23 February 2011; accepted 2 March 2011

Background: To evaluate the outcome of breast cancer patients after locoregional recurrence (LRR) according to tumor biological features evaluated at first diagnosis and at the time of recurrence.

Patients and methods: We collected information on all consecutive breast cancer patients operated at the European Institute of Oncology between 1994 and 2005. The tumor characteristics and subsequent outcome of patients who experienced LRR were analyzed.

Results: Two hundred and seventy nine patients with LRR were identified, 197 and 82 patients with local and regional recurrence respectively. The overall discordance rate between primary cancer and LRR was $9 \%$ for estrogen receptor expression, 22\% for progesterone receptor and $4 \%$ for human epidermal growth factor receptor 2. For patients with regional recurrence, the risk of distant metastasis was significantly higher compared with local relapse in case of late recurrence (hazard ratio $[\mathrm{HR}]=2.76 ; 95 \% \mathrm{Cl} 1.31-5.85$ ). Patients with triplenegative breast cancer at LRR experienced a higher risk of subsequent relapse (HR 2.87 [1.67-4.91]) and death (HR 2.00 [1.25-3.19]).

Conclusion: LRR correlates with a high risk of subsequent events and death in particular in patients with triple-negative subtype.

Key words: breast, cancer, locoregional, relapse

\section{introduction}

Locoregional recurrences (LRR) may occur in a significant proportion of patients after primary therapy despite careful local treatment and systemic adjuvant therapies and or radiotherapy [1-3]. Patients who are free of overt metastases after initial local and regional treatment eventually die of recurrence of distant disease. In particular, breast cancer patients with LRR have been found to have 5-year disease-free survival (DFS) rates of $13 \%-37 \%$ and overall survival (OS) rates of $21 \%-50 \%$ [4-6], respectively.

A 10 -year estimated OS rate of up to $26 \%$ has been reported, indicating that long-term reappearance of disease should be taken into account [5, 7]. Moreover, the outcome is worse after regional recurrence compared with local [8].

A better description of relapse patterns may improve patient outcome by permitting a better understanding of patients'

${ }^{*}$ Correspondence to: Dr E. Montagna, Department of Medicine, Division of Medica Oncology, Research Unit of Medical Senology, European Institute of Oncology, Via Ripamonti 435, 20141 Milan, Italy. Tel: +39-02-57489439; Fax; +39-02-574829212;

E-mail: emilia.montagna@ieo.it subgroup-specific risk, which could lead to targeted therapeutic approaches. Recently, different subtypes of breast cancer have been identified based on gene expression profiles and immunohistochemical evaluations with specific responsiveness to treatments and outcome [9-11]. In particular, Hugh et al. [12] reported that patients with ER (estrogen receptor)negative tumors (both triple negative and HER2 [human epidermal growth factor receptor 2] positive) showed a better response to adjuvant taxane-containing chemotherapy than to chemotherapy that does not contain taxane. Moreover, the magnitude of the effect for the classical combination chemotherapy with cyclophosphamide, methotrexate and fluorouracil regimen was reported to be larger in patients with the triple-negative subtype [13].

Given, the paramount importance of using targeted therapies wherever possible for adjuvant treatment, timely, accurate and reliable histopathologic assessment that identifies and quantifies the target is essential. We therefore aimed to evaluate the influence of LRR on disease outcome in a large series of patients who were homogeneously diagnosed and treated in a single institution according to tumor biological features evaluated at first diagnosis and at the time of recurrence. 


\section{patients and methods}

\section{patients}

We reviewed information on 14733 consecutive breast cancer patients whose definitive breast cancer surgery was carried out at the European Institute of Oncology between 1994 and 2005. Data on the patient's medical history, concurrent diseases, surgery, pathological evaluation, and results of staging procedures (blood chemistry, hematologic values, bone scan, chest film and upper abdominal ultrasound examination) were required. This report concerns patients who subsequently developed LRR as first event. We excluded patients who initially presented with bilateral breast tumor or who received neoadjuvant treatments. Following surgery, all cases were discussed during the weekly multidisciplinary meeting attended by surgeons, medical oncologists, radiation oncologists and pathologists. The decision for adjuvant systemic treatment was made on the basis of biological features, staging, treatment previously received and comorbidities. Women were usually followed up by physical examination every 6 months and mammography with or without breast ultrasound (US) annually. In symptomatic cases or when clinically indicated, bone scan, chest X-ray, liver US or computed tomography scan were carried out.

We identified patients who experienced LRR as first event without evidence of distant metastasis. A recurrence was categorized as local (in the ipsilateral breast in case of primary breast-conserving treatment, operation scar, or chest wall or skin) or regional (in the axillary lymph nodes or the supraclavicular region internal mammary or supraclavicular or infraclavicular nodes).

Patients with LRR were treated according to standard multidisciplinary protocols. All patients received adequate local treatment using surgery, radiotherapy or both. The selection of systemic treatment was individually based upon indicators of responsiveness to treatment (ER, PgR [progesterone receptor] and HER2 expression of the tumor), previous treatment and indicators of risk. According to the recent classification of breast cancer, we identified four immunohistochemically defined tumor subtypes: triple negative (ER, PgR and HER2 negative), HER2 positive (HER2 positive, ER and PgR negative) and endocrine-responsive: luminal A (with ER and/or PgR > 1\%, Ki-67 < 14\% and HER2 negative) and luminal B (with ER and/or PgR > 1\%and Ki- $67 \geq 14 \%$ or HER2 positive or both). Patients with the luminal A subtype were commonly candidate to endocrine therapies selected upon previous endocrine agents received. Patients with the luminal B subtype also received short-term (four courses) chemotherapy (e.g. anthracyclines- or taxanes-containing chemotherapy), whereas those with triple-negative breast cancer were candidate to cisplatincontaining chemotherapy or alkylating-containing chemotherapy for 4-6 months. In case of HER2 overexpression and/or amplification, trastuzumab for 12 months was prescribed starting from 2005.

\section{pathology and immunohistochemistry}

In this single institution study, all patients had pathological evaluation carried out in the European Institute of Oncology both at primary surgery and at LRR.

Immunostaining for the localization of ER and PgR, HER2 protein and Ki-67 antigen was carried out on consecutive tissue sections from the tumor-containing blocks, as previously reported [14]. The following primary antibodies were used: the monoclonal antibody (MAb) to ER (Dako, Glostrup, Denmark at 1/100 dilution), the MAb to PgR (Dako at 1/800 dilution), the MIB-1 MAb to the Ki-67 antigen (Immunotech, Marseille, France, 1/1200) and the polyclonal antiserum (Dako at 1/3200 dilution) to the HER2 protein.

Only nuclear reactivity was taken into account for ER, PgR and Ki-67 antigen, whereas only an intense and complete membrane staining in $>10 \%$ of the tumor cells qualified for HER2 overexpression (3+). The results for ER, PgR and Ki-67 were recorded as the percentage of immunoreactive cells observed among at least 2000 neoplastic cells. The value Ki-67 labeling index was divided into low $(<14 \%)$ and high $(\geq 14 \%)$ [11].

\section{statistical analysis}

The Pearson $\chi^{2}$ test was used to compare the distribution of clinical and pathological variables between patients with local and regional recurrence as first event.

The end points evaluated were progression-free survival (PFS), OS, cumulative incidence of LRR (CI-LR) and distant recurrence (CI-D), all measured from the date of LRR.

PFS was defined as the length of time from the date of LRR to any subsequent relapse (including ipsilateral breast recurrence, contralateral breast cancer, appearance of a second primary cancer) or death, whichever occurred first.

OS was defined as the time from the date of LRR until the date of death (from any cause).

The PFS and OS functions were estimated using the Kaplan-Meier method. The log-rank test was used to assess differences between patients with local versus regional recurrence as first event.

The CI-LR and CI-D were defined as the length of time from the date of first LRR to a second LRR and distant metastases, respectively.

The CI-LR and CI-D curves functions were estimated according to methods described by Kalbfleisch and Prentice [15], taking into account the competing causes of recurrence. The Gray's test was used to assess cumulative incidence differences between patients with local and regional recurrence as first event [16].

The prognostic impact of several factors on PFS, OS and cause-specific hazard was evaluated using multivariable Cox proportional hazards regression models and expressed as hazard ratio (HR) with 95\% confidence interval (CI) [17]. All analyses were carried out with the SAS software (SAS Institute, Cary, $\mathrm{NC}$ ) and the R software (http://cran.r-project.org/) with the cmprsk package developed by Gray (http://biowww.dfci.harvard.edu/ $\sim$ gray). All reported $P$-values are two-sided.

\section{results}

A total of 14733 consecutive patients with breast cancer who had definitive surgery for breast cancer, referred for interdisciplinary evaluation between 1994 and 2005 and included in a prospective quality-controlled database, were analyzed. We excluded patients who presented with metastatic disease $(n=364)$, other previous primary tumor $(n=400)$, previous contralateral breast cancer $(n=420)$, recurrent disease at presentation $(n=683)$, primary chemotherapy $(n=1341)$ and male breast cancer $(n=73)$. On the remaining 11452 patients, a total of 279 patients with LRR (197 and 82 patients with local and regional recurrence) were identified.

At the time of the primary cancer of study population, $74 \%$ and $58 \%$ of patients with subsequent local and regional recurrence respectively had undergone conservative surgery. Regional recurrence occurred more frequently than local recurrence (31\% versus $26 \%$ ) among patients who presented with four or more positive lymph nodes at primary surgery.

After primary surgery, $12 \%$ of study patients didn't receive any adjuvant therapy, 63\% received endocrine therapy, $61 \%$ chemotherapy ( $36 \%$ received both), while adjuvant standard radiotherapy or intraoperative electron beam (ELIOT) radiotherapy was used in $62 \%$ of cases. Only one patient received trastuzumab in the adjuvant setting. 
Table 1 reports the timing and clinical and biological features at LRR and treatment received. Twelve percent and $16 \%$ of patients with local and regional recurrence had a time to LRR $<1$ year. The time to LRR from primary breast cancer was from 2 to 4 years in the $32 \%$ of the local recurrence group and in the $38 \%$ of regional recurrence group. The median time to LRR was 2.6 years (range $0.3-9.3$ years). Only $12 \%$ and $8 \%$ in the

Table 1. Characteristics at first locoregional recurrence and treatment received after recurrence, by type of recurrence

\begin{tabular}{lccc} 
& $\begin{array}{l}\text { Local recurrence } \\
(n=197) \\
n(\%)\end{array}$ & $\begin{array}{l}\text { Regional recurrence } \\
(n=82) \\
n(\%)\end{array}$ & $P^{\mathrm{a}}$ \\
$\begin{array}{l}\text { Year of recurrence } \\
<2001\end{array}$ & $32(16.2)$ & $12(14.6)$ & 0.40 \\
$2001-2003$ & $65(33.0)$ & $34(41.5)$ & \\
$2004-2005$ & $100(50.8)$ & $36(43.9)$ & 0.34 \\
Age (years) & & & \\
$<35$ & $10(5.1)$ & $2(2.4)$ & \\
$35-50$ & $78(39.6)$ & $29(35.4)$ & \\
$51-65$ & $67(34.0)$ & $26(31.7)$ & \\
$>65$ & $42(21.3)$ & $25(30.5)$ & \\
Time to recurrence (years) & & & \\
$<1$ & $24(12.2)$ & $13(15.9)$ & \\
$1-2$ & $46(23.4)$ & $25(30.5)$ & \\
$2-4$ & $64(32.5)$ & $31(37.8)$ & \\
$>4$ & $63(32.0)$ & $13(15.9)$ & \\
Breast cancer subtype ${ }^{\text {b.c }}$ & & & \\
Luminal A & $30(15.2)$ & $8(9.8)$ & \\
Luminal B ${ }^{\text {d }}$ & $99(50.3)$ & $44(53.7)$ & \\
HER2 positive & $26(13.2)$ & $12(14.6)$ & \\
Triple negative & $32(16.2)$ & $13(15.9)$ & \\
Unknown & $10(5.1)$ & $5(6.1)$ & \\
CT/HT & & & \\
Nil & $25(12.6)$ & $7(8.5)$ & \\
HT & $101(51.2)$ & $33(40.2)$ & \\
CT & $53(26.9)$ & $24(29.2)$ & \\
CT-HT & $18(9.1)$ & $18(21.9)$ & \\
\hline
\end{tabular}

${ }^{a}$ Chi-square test comparing frequencies between local recurrence group and regional recurrence group.

${ }^{b}$ Luminal A: $(\mathrm{ER}>0$ or PgR $>0)$ and $(\mathrm{Ki}-67<14 \%)$ and (HER2 negative); Luminal B: (ER $>0$ or PgR $>0$ ) and ([Ki-67 $\geq 14 \%]$ or [HER2 positive]); HER2 positive: ( $E R=0$ and $\mathrm{PgR}=0$ ) and (HER2 positive); Triple negative (ER negative and PgR negative) and (HER2 negative).

'If any biological variable was unknown at locoregional recurrence, the value at primary breast cancer was used. The value of Ki-67 expression was unknown at locoregional recurrence in 12 luminal A, 48 luminal B, 9 HER2-positive and 15 triple-negative breast cancer subtypes. The HER2 status was unknown at locoregional recurrence in one HER2-positive and two triple-negative breast cancer subtypes.

${ }^{\mathrm{d}}$ Among luminal B, 34 patients were HER2 positive (22 and 12 in local and regional recurrence group, respectively). Among these, eight patients received trastuzumab after recurrence (three and five in the local and regional recurrence group, respectively).

${ }^{\mathrm{e}}$ Twenty one patients received trastuzumab after recurrence ( 13 and 8 in the local and regional recurrence group, respectively).

CT, chemotherapy; ER, estrogen receptor; HER2, human epidermal growth factor receptor 2; HT, hormonal therapy; PgR, progesterone receptor. local and regional group respectively did not receive an adjuvant systemic therapy.

Table 2 shows the comparison of biological features and subtypes between primary cancer and LRR. We reported an overall discordance rate of 9\% for ER expression, $22 \%$ for PgR expression, 19\% for Ki-67, 4\% for HER2 and 22\% for subtypes. For the hormonal receptor status, more frequently we reported a loss of expression in the LRR compared with primary tumor. In particular, 18 breast cancer cases with ER positive became negative at LRR, while 46 tumors with PgR positive became negative at relapse.

Median follow up after LRR was 5.9 years (range 0.9-12.2 years). The Kaplan-Meier curves for PFS and OS according to the type of relapse (local or regional) are displayed in Figure 1A and B. The 5-year PFS and OS were $45 \%$ and $71 \%$ in the local recurrence group compared wirh $34 \%$ and $58 \%$ in the regional recurrence group ( $P=0.052$ for $\mathrm{PFS}$ and $P=0.063$ for OS). Figure $1 \mathrm{C}$ and $\mathrm{D}$ shows the CI-LR alone and CI-D. The 5-year CI-LR and CI-D were $25 \%$ and $21 \%$ in the local recurrence group compared with 5-year CI-LR and CI-D of $21 \%$ and $36 \%$ in the regional recurrence group. ( $P=0.39$ for CI-LR and $P=0.013$ for CI-D).

Moreover, we evaluated the impact of early recurrence ( $<2$ years) on CI-D in both local and regional groups. For patients with local recurrence, the CI-D was significantly higher in case of early recurrence compared with late relapse; in the meanwhile, patients with regional recurrence continue to have higher risk of distant metastasis despite to time of recurrence (interaction $P$-value $=0.003$ ) (Figure $2 \mathrm{~A}$ and $\mathrm{B}$ ).

The Kaplan-Meier curves for PFS and OS according to the subtypes at recurrence are shown in Figure 3. We reported the highest incidence of subsequent event among those patients presenting with the triple-negative subtype. In particular, after 2 years the PFS was $36 \%$ (95\% CI $22 \%$ to $51 \%$ ) and OS $60 \%$ (95\% CI $46 \%$ to $74 \%$ ).

At the time of the study analyses, 103 patients were free of disease, 73 patients experienced new LRR and 74 patients experienced distant recurrence with bone involvement in 36 patients. The 5 -year CI of bone involvement was $8 \%$ in patients with local recurrence and $19 \%$ in patients with regional recurrence $(P=0.04)$.

Moreover, for patients with local recurrence, we evaluated the outcome according to the type of surgery (quadrantectomy or mastectomy) at primary cancer. No differences were observed.

\section{multivariate analysis}

The independent impact on time to event following LRR was assessed for various features of the primary cancer and type of LRR. The results are displayed in Table 3.

For patients with regional recurrence, the risk of distant metastasis was significantly higher compared with local relapse in case of late recurrence (HR 2.76; 95\% CI 1.31-5.85).

The presence of vascular invasion (HR 1.59; 95\% CI 0.96-2.65 for PFS, HR 1.61; 95\% CI 1.09-2.37 for OS) and high grade at primary cancer (HR 1.76; 95\% CI 1.06-2.94 for PFS, HR 1.40; 95\% CI 0.95-2.08 for OS) were associated with worse PFS and OS. Triple-negative breast cancer subtype at 
Table 2. Qualitative changes in receptor expression (ER and PgR), Ki-67 and HER2 status between primary breast cancer and locoregional recurrence. For ER and PgR, positive status was defined as $\geq 1 \%$, high Ki- 67 was defined as $\geq 14 \%$, HER2-positive status was defined as $>3$

Locoregional recurrence

ER

$\begin{array}{ll}\text { Primary tumor } & \text { Negative }(n) \\ \text { Negative } & 70 \\ \text { Positive } & 18 \\ \text { Total } & 88\end{array}$

Positive $(n)$

7

184

191

Overall discordance rate ( $95 \% \mathrm{CI}): 9.0 \%(5.9 \%$ to $12.9 \%)$

PgR

Primary tumor Negative $(n)$

Negative 105

Positive 46

16

Positive $(n)$

$\begin{array}{rr}16 & 121 \\ 112 & 158 \\ 128 & 279\end{array}$

Total

151

Overall discordance rate ( $95 \% \mathrm{CI}): 22.2 \%$ ( $17.5 \%$ to $27.6 \%)$

HER2 status ${ }^{\mathrm{a}}$

Primary tumor

Negative $(n)$

Negative

119

Positive

3

Total

122

Positive (n)

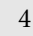

48

Overall discordance rate $(95 \% \mathrm{CI}): 4.0 \%$ (1.6\% to $8.1 \%)$

$\mathrm{Ki}-67^{\mathrm{b}}$

Primary tumor Low $(n)$

Low 12

High 21

Total 33

\section{High $(n)$}

$13 \quad 25$

$135-156$

$148-181$

Overall discordance rate ( $95 \% \mathrm{CI}): 18.8 \%$ ( $13.4 \%$ to $25.2 \%)$

Breast cancer subtype

Primary tumor

Luminal A $(n)$

Luminal A

Luminal B

HER2 positive

Triple negative

Total

7
8
0
0
15

Overall discordance rate ( $95 \% \mathrm{CI}): 22.1 \%$ ( $15.4 \%$ to $30.0 \%)$

Luminal B $(n)$
5
48
1
0
54

HER2 positive $(n)$
1
9
1
25
36

Triple negative $(n)$

${ }^{a}$ One hundred and five patients with missing value at primary breast cancer or at locoregional recurrence were not considered.

${ }^{b}$ Ninety eight patients with missing value at primary breast cancer or at locoregional recurrence were not considered.

${ }^{c}$ One hundred and forty eight patients with missing value at primary breast cancer or at locoregional recurrence were not considered.

CI, confidence interval; ER, estrogen receptor; HER2, human epidermal growth factor receptor 2; PgR, progesterone receptor.

recurrence was associated with increased risk of subsequent event and death (HR 2.87; 95\% CI 1.67-4.91 for PFS, HR 2.00; 95\% CI 1.25-3.19 for OS).

\section{discussion}

In this study, we evaluated the patterns of subsequent recurrence among the 279 patients who had a first LRR. Our focus was on subgroups of patients with higher risk of further with the possibility of investigating targeted treatments. All the patients included in the present analysis had a histological evaluation both at first diagnosis and at recurrence at the same institution. Thus, the evaluation of biological features carried out by the same group of pathologist in all patients is a major strength of the current analysis.

Our study confirms the negative prognostic impact of LRR in breast cancer patients. The 5-year PFS and OS were $45 \%$ and $71 \%$ in the local recurrence group and $34 \%$ and $58 \%$ in the regional recurrence group. Wapnir et al. [8] reported that after local recurrence the proportion of patients who were free of distant disease at 5 years was $51 \%$ and the proportion of patients surviving was $60 \%$. The 5 -year distant DFS and OS were $19 \%$ and $24 \%$ in patients who experienced regional recurrence.

The most relevant observation concerning the patterns of relapse was the highest incidence of subsequent event among those patients presenting with the triple-negative subtype (Figure 3). In fact at the multivariate analyses, we reported that the triple-negative subtype at recurrence was significantly associated with worse prognosis in terms of PFS and OS. Moreover, patients with triple-negative tumors had a higher early incidence of event. In particular, after 2 years the PFS was $36 \%$ (95\% CI $22 \%$ to $51 \%$ ) and OS, $60 \%$ ( $46 \%$ to $74 \%$ ). It is noteworthy that among the 45 patients with triple-negative subtype, $33(73 \%)$ of the patients received adjuvant chemotherapy and 29 (64\%) adjuvant radiotherapy. 
A
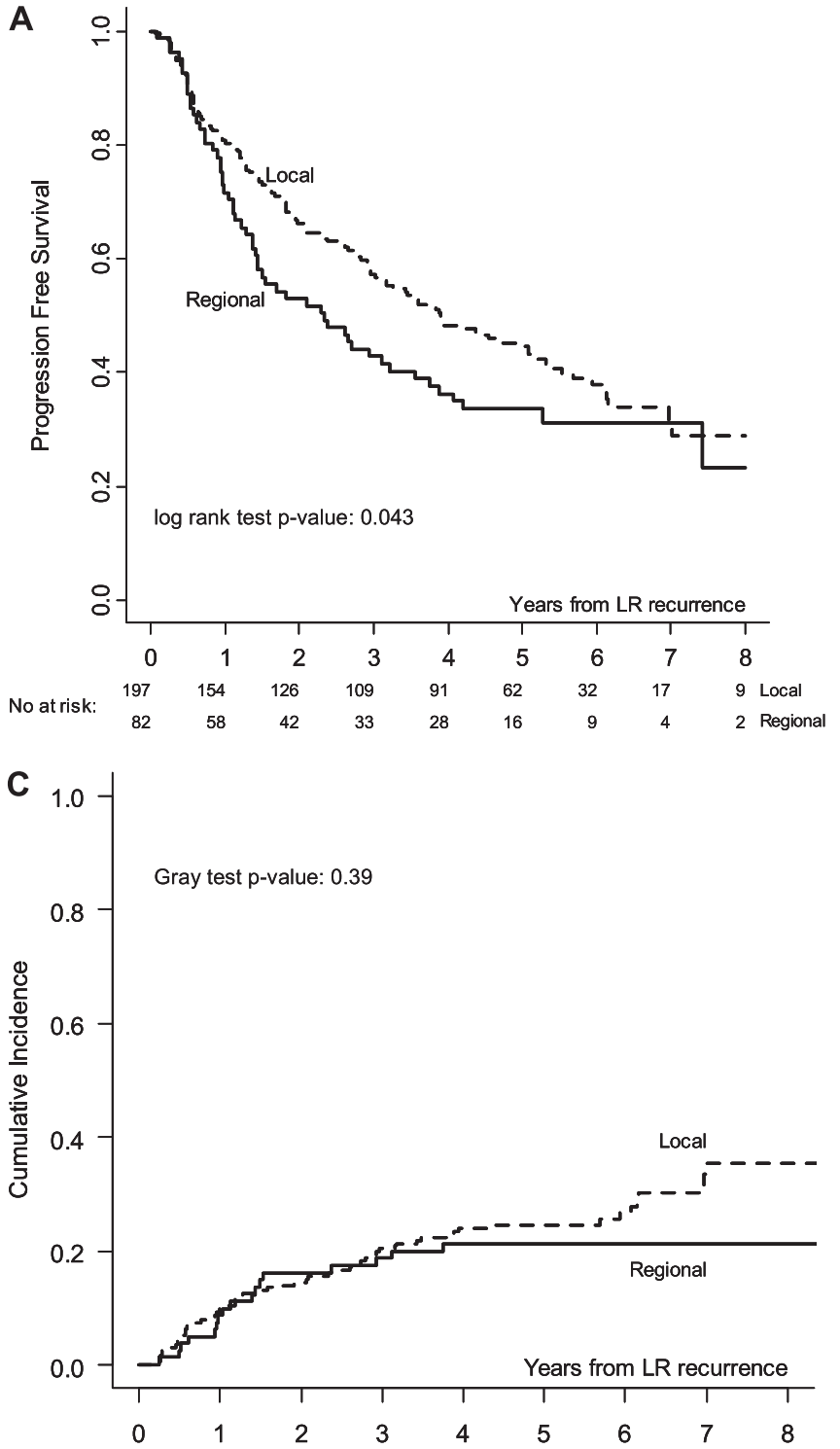

B
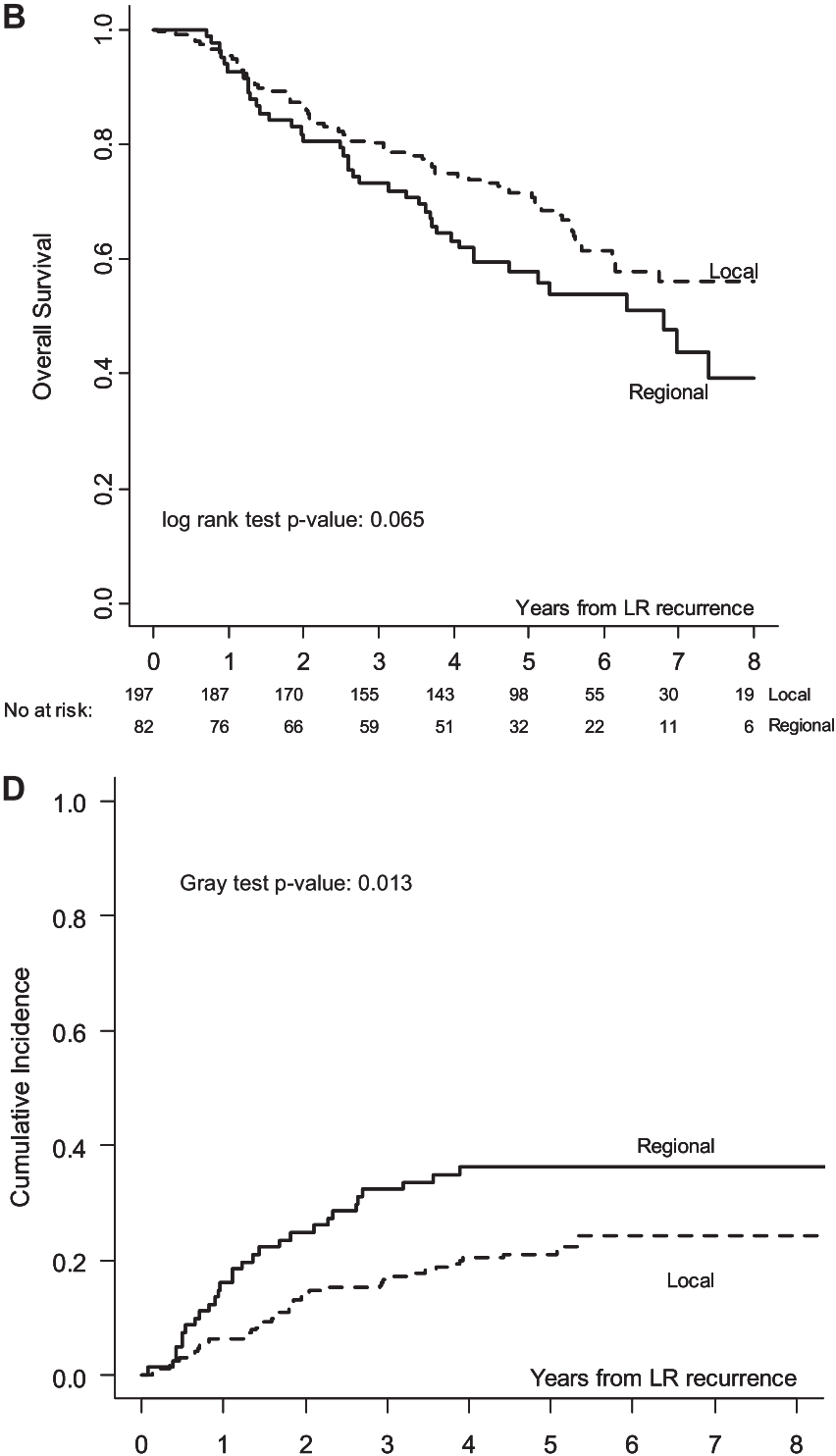

Figure 1. Progression-free survival (A), overall survival (B), cumulative incidence of locoregional event (C) and distant event (D) after first locoregional recurrence, by type of first recurrence.

We also compared pathological features of primary tumor and LRR and we observed that the expression of biological parameters changed between primary and recurrence (Table 2). We reported an overall discordance rate of $9 \%$ for ER expression, 22\% for PgR expression (predominantly changed from positive to negative) $19 \%$ for Ki-67 (mainly increased) and $4 \%$ for HER2. In particular, 18 breast cancers with ER positive became negative at LRR, while 46 tumors with PgR positive became negative at relapse. Due to the low number of cases, it is however difficult to investigate the predictive or prognostic value of a changed receptor status. The mechanisms of change of hormone receptor and HER2 expression have not been explicitly studied. Adjuvant chemotherapy and hormonal therapy may eliminate specific subclones of tumor cells, selecting others that dominate in the recurrent disease [18]. Receptor status may also depend on the analytical method used and by variation in tissue processing and fixation.
Lower et al. [19] analyzed the concordance on HER2 status between primary cancer and metastasis in 382 breast cancer patients. The authors reported a higher rate of discordance in $33.2 \%$ of cases with $23.6 \%$ changed from positive to negative, while $9.6 \%$ changed from negative to positive [19]. Other studies have reported hormone receptor status discordance rates of $21 \%-28 \%$ (predominantly loss of hormonal receptors) between primary and breast relapse $[20,21]$. In the studies above mentioned, most comparisons regarded primary with distant metastatic sites and very few patients with LRR were included. In the study of Carreno et al. [22], the comparison of primary tumor and LRR was done in 25 patients reporting a substantial concordance in the expression of hormonal receptor between primary tumor and LRR.

In our study, patients who experienced regional recurrence had a worse prognosis than those with local relapse. Patients with regional recurrence continue to have higher risk of distant metastasis irrespective of time of relapse. Conversely, in 

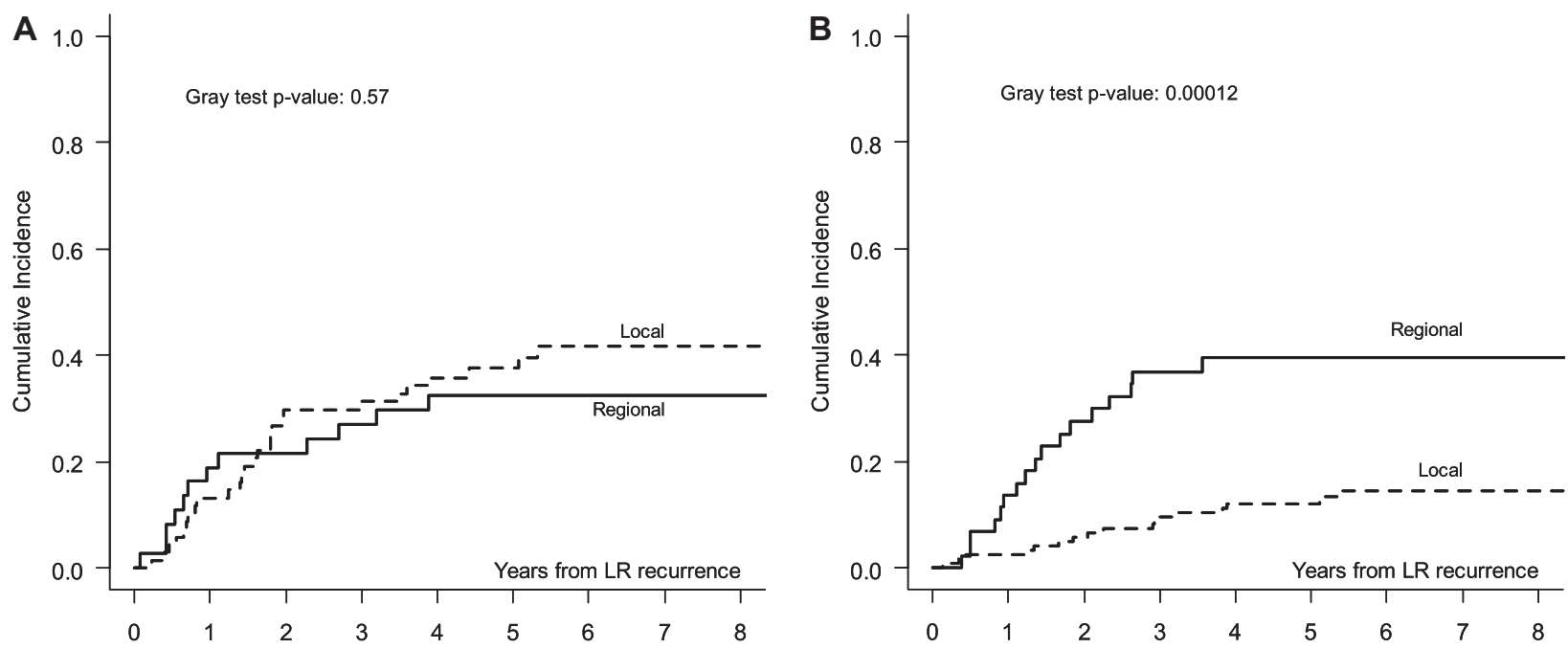

Figure 2. Cumulative incidence of distant event after first locoregional recurrence, by type of first recurrence, in patients with time to first recurrence $<2$ years $(\mathrm{A})$ and $>2$ years $(\mathrm{B})$.
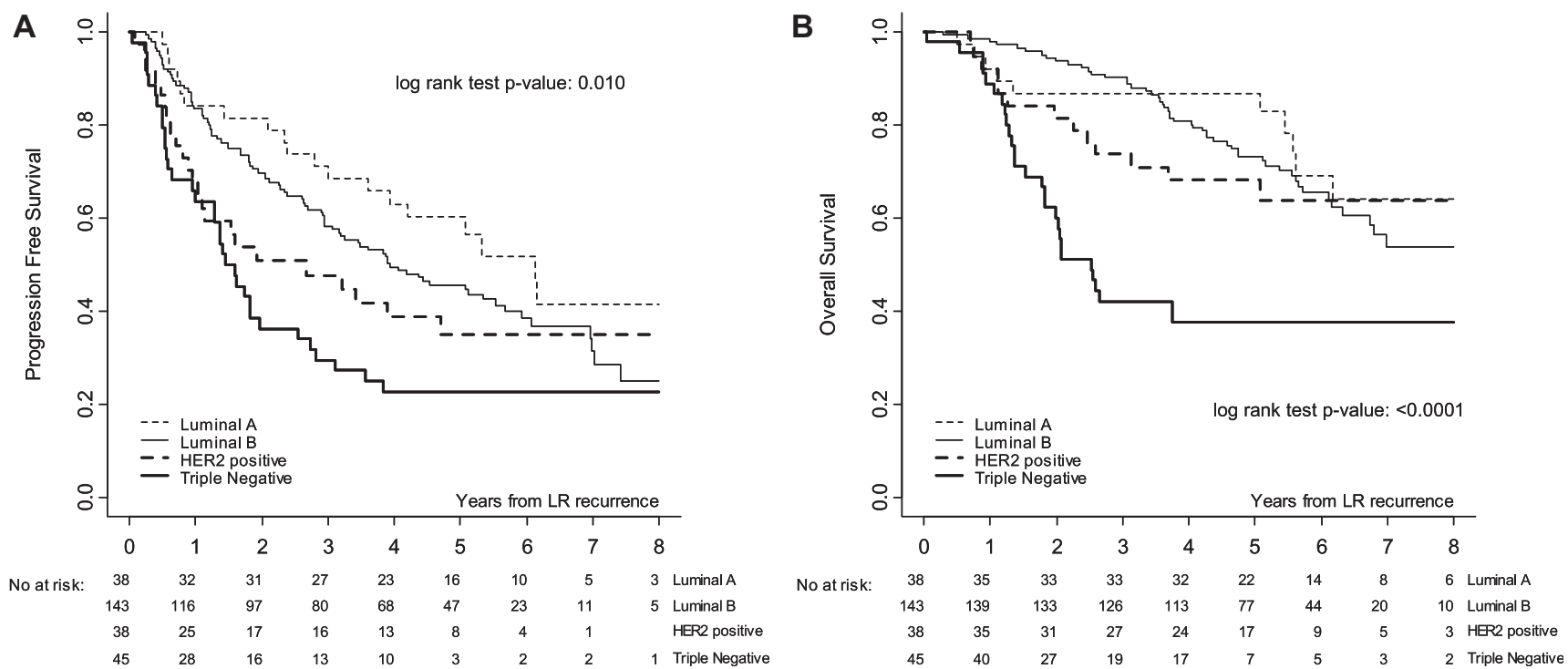

Figure 3. Progression-free survival (A) and overall survival (B) after first LRR (locoregional recurrence), by subtypes evaluated at LRR. Note: If any biological variable was missing at locoregional recurrence, the value at primary breast cancer was used. The value of Ki-67 expression was missing at locoregional recurrence in 12 luminal A, 48 luminal B, 9 HER2-positive and 15 triple-negative breast cancer subtypes. The HER2 status was missing at locoregional recurrence in one HER2-positive and two triple-negative breast cancer subtypes.

patients with local recurrence a dismal prognosis was observed only among those that experienced an early relapse. Other studies also reported better a good outcome for patients with late recurrence [23]. In case of local recurrence in fact, the late relapse could suggest a second primary breast cancer more than a relapse of the previous tumor.

Regarding the patterns of distant metastases, we reported a high incidence of subsequent bone involvement. In particular, $48 \%$ of patients who experienced distant disease presented with bone metastasis. A high incidence of subsequent relapse in bone was also found in the retrospective analyses of pattern of recurrence in patients included in the adjuvant studies of International Breast Cancer Study Group trial between 1978 and 1993 [24]. The authors reported a 10-year cumulative incidence of $36.7 \%$ with regard to skeletal involvement at any time. The analysis of pattern of distant relapse in patients who experienced LRR could suggest the implementation of strategy to reduce the rate of bone involvement in patients with LRR with bisphosphonates, which might reduce the relapse of growth factors from microfoci of bone destruction, thereby reducing bone absorption and decreasing stimuli of micrometastatic breast cancer $[25,26]$.

The finding that patients with LRR remain at substantial risk of relapse, was observed despite hormonal and/or chemotherapy program (Table 1), which might have interfered with the outcome. The benefit of hormonal therapy after LRR was reported. The trial by Waeber et al. [27], compared tamoxifen with observation in 167 patients who underwent radical 
Table 3. Multivariable Cox regression analysis for PFS, OS, CSH-LR and CSH-D recurrence, after first locoregional recurrence

\begin{tabular}{|c|c|c|c|c|}
\hline & $\begin{array}{l}\text { PFS } \\
\text { HR (95\% CI) }\end{array}$ & $\begin{array}{l}\text { OS } \\
\text { HR }(95 \% \text { CI })\end{array}$ & $\begin{array}{l}\text { CSH-LR } \\
\text { HR ( } 95 \% \text { CI) }\end{array}$ & $\begin{array}{l}\text { CSH-D } \\
\text { HR ( } 95 \% \text { CI) }\end{array}$ \\
\hline \multicolumn{5}{|c|}{ Time to LRR >2 years } \\
\hline \multicolumn{5}{|c|}{ Type of recurrence } \\
\hline Local & Reference category & & & \\
\hline Regional & $1.50(0.76-2.98)$ & $1.49(0.90-2.46)$ & $0.76(0.31-1.87)$ & $2.76(1.31-5.85)$ \\
\hline \multicolumn{5}{|c|}{ Time to LRR $<2$ years } \\
\hline \multicolumn{5}{|c|}{ Type of recurrence } \\
\hline Local & Reference category & & & \\
\hline Regional & $1.01(0.53-1.95)$ & $0.97(0.55-1.71)$ & $0.65(0.23-1.85)$ & $1.10(0.53-2.29)$ \\
\hline \multicolumn{5}{|l|}{ Age at LRR } \\
\hline$>50$ years & Reference category & & & \\
\hline$\leq 50$ years & $0.64(0.39-1.03)$ & $0.81(0.56-1.17)$ & $0.93(0.52-1.65)$ & $0.84(0.48-1.46)$ \\
\hline \multicolumn{5}{|c|}{ Breast cancer subtype (at LRR) $)^{\mathrm{a}, \mathrm{b}}$} \\
\hline Luminal A/B & Reference category & & & \\
\hline HER2 positive & $0.79(0.38-1.66)$ & $1.34(0.78-2.30)$ & $1.89(0.83-4.31)$ & $0.71(0.30-1.64)$ \\
\hline Triple negative & $2.87(1.67-4.91)$ & $2.00(1.25-3.19)$ & $1.89(0.87-4.13)$ & $1.45(0.74-2.83)$ \\
\hline \multicolumn{5}{|c|}{ Grade (at primary breast cancer) } \\
\hline $1-2$ & Reference category & & & \\
\hline 3 & $1.76(1.06-2.94)$ & $1.40(0.95-2.08)$ & $1.11(0.60-2.08)$ & $2.36(1.31-4.26)$ \\
\hline \multicolumn{5}{|c|}{ pT (at primary breast cancer) } \\
\hline pT1 & Reference category & & & \\
\hline pT2-4 & $1.00(0.60-1.65)$ & $1.36(0.94-1.98)$ & $1.18(0.67-2.10)$ & $1.23(0.70-2.16)$ \\
\hline \multicolumn{5}{|c|}{ Nodal status (at primary breast cancer) } \\
\hline Negative & Reference category & & & \\
\hline Positive & $1.24(0.71-2.15)$ & $1.19(0.79-1.79)$ & $1.04(0.55-1.99)$ & $1.45(0.79-2.69)$ \\
\hline \multicolumn{5}{|c|}{ PVI (at primary breast cancer) } \\
\hline Absent & Reference category & & & \\
\hline Present & $1.59(0.96-2.65)$ & $1.61(1.09-2.37)$ & $1.83(0.98-3.40)$ & $1.73(0.98-3.04)$ \\
\hline
\end{tabular}

${ }^{a}$ Luminal A: $(\mathrm{ER}>0$ or PgR $>0)$ and $(\mathrm{Ki}-67<14 \%)$ and (HER2 negative); Luminal B: (ER > 0 or PgR >0) and ([Ki-67 $\left.\geq 14 \%\right]$ or $[$ HER2 positive]); HER2 positive: (ER $=0$ and $\mathrm{PgR}=0$ ) and (HER2 positive); Triple negative (ER negative and PgR negative) and (HER2 negative).

${ }^{\mathrm{b}}$ If any biological variable was missing at locoregional recurrence, the value at primary breast cancer was used. The value of Ki- 67 expression was missing at locoregional recurrence in 12 luminal A, 48 luminal B, 9 HER2-positive and 15 triple-negative breast cancer subtypes. The HER2 status was missing at locoregional recurrence in one HER2-positive and two triple-negative breast cancer subtypes.

CI, confidence interval; CSH-LR, cause-specific hazard for locoregional; CSH-D, cause-specific hazard for distant; ER, estrogen receptor; HR, hazard ratio; LRR, locoregional recurrence; OS, overall survival; PFS, progression-free survival; PgR, progesterone receptor; PVI, peritumoral vascular invasion.

surgery and radiotherapy for post mastectomy LRR. After a median follow up of 11 years, tamoxifen therapy significantly improves PFS. This beneficial effect does not translate into a detectable OS advantage. On the other hand, the utility of chemotherapy for women who experience an LRR remains an open question. An international collaborative trial BIG 1-02/IBCSG 27-02/NSABP B-37 is currently being conducted to determine the effectiveness of cytotoxic therapy for these patients.

In conclusion, we demonstrated that LRR correlate with high risk of subsequent events and death in particular within the triple-negative subtype. New chemotherapy regimens or combinations should be explored in this cohort of patients with poor outcome. Moreover, the variation of biologic parameters between primary tumor and LRR and the related clinical and therapeutic implications, support a histopathologic reevaluation of the disease at the time of LRR.

\section{disclosure}

The authors declare no conflicts of interest.

\section{references}

1. Fisher $B$, Anderson $S$, Bryant $J$ et al. Twenty year follow-up of a randomized trial comparing total mastectomy, lumpectomy, and lumpectomy plus irradiation for the treatment of invasive breast cancer. N Engl J Med 2002; 347: 1233-1241.

2. Veronesi $U$, Cascinelli N, Mariani $L$ et al. Twenty-year follow-up of a randomized study comparing breast-conserving surgery with radical mastectomy for early breast cancer. N Engl J Med 2002; 347: 1227-1232.

3. Blichert-Toft M, Nielsen M, Düring M et al. Long-term results of breast conserving surgery vs. mastectomy for early stage invasive breast cancer: 20-year follow-up of the Danish randomized DBCG-82TM protocol. Acta Oncol 2008; 47(4): 672-681.

4. Valagussa P, Tess JD, Rossi A et al. Adjuvant CMF effect on site of first recurrence, and appropriate follow-up intervals, in operable breast cancer with positive axillary nodes. Breast Cancer Res Treat 1981 1: $349-356$

5. Goldhirsch A, Gelber RD, Price KN et al. Effect of systemic adjuvant treatment on first site of breast cancer relapse. Lancet 1994; 343: 377-381.

6. Whelan $T$, Clark R, Roberts $R$ et al. Ipsilateral breast tumor recurrence postlumpectomy is predictive of subsequent mortality: results from a randomized trial. Int J Radiat Oncol Biol Phys 1994; 30: 11-16. 
7. Kamby $K$, Senegelov L. Pattern of dissemination and survival following isolated locoregional recurrence of breast cancer: a prospective study with more than 10 years of followup. Breast Cancer Res Treat 1997; 45: 181-192.

8. Wapnir IL, Anderson SJ, Mamounas EP et al. Prognosis after ipsilateral breast tumor recurrence and locoregional recurrences in five National Surgical Adjuvant Breast and Bowel Project node-positive adjuvant breast cancer trials. J Clin Oncol 2006; 24: 2028-2037.

9. Perou CM, Sorlie T, Eisen MB et al. Molecular portraits of human breast tumors. Nature 2000; 406(6797): 747-752.

10. de Ronde JJ, Hannemann J, Halfwerk $H$ et al. Concordance of clinical and molecular breast cancer subtyping in the context of preoperative chemotherapy response. Breast Cancer Res Treat 2010; 119(1): 119-126.

11. Cheang MC, Chia SK, Voduc D et al. Ki67 index, HER2 status, and prognosis of patients with luminal B breast cancer. J Natl Cancer Inst 2009; 101(10): 736-750.

12. Hugh J, Hanson J, Cheang MC et al. Breast cancer subtypes and response to docetaxel in node positive breast cancer: use of an immunohistochemical definition in the BCIRG 001 trial. J Clin Oncol 2009; 27: 1168-1176.

13. Colleoni $\mathrm{M}$, Cole BF, Viale $\mathrm{G}$ et al. Classical cyclophosphamide, methotrexate, and fluorouracil chemotherapy is more effective in triple-negative, node-negative breast cancer: results from two randomized trials of adjuvant chemoendocrine therapy for node-negative breast cancer. J Clin Oncol 2010; 28(18): 2966-2973.

14. Colleoni $\mathrm{M}$, Orvieto $\mathrm{E}$, Nolè $\mathrm{F}$ et al. Prediction of response to primary chemotherapy for operable breast cancer. Eur J Cancer 1999; 35: 574-579.

15. Kalbfleisch JD, Prentice RL. The Statistical Analysis of Failure Time Data. Hoboken, NJ: Wiley \& Sons Ltd 1980.

16. Gray RJ. A class of K-sample tests for comparing the cumulative incidence of a competing risk. Ann Stat 1988; 16: 1141-1154.

17. Cox D. R: regression models and life tables (with discussion). J R Stat Soc 1972; B34: $187-220$.
18. Lee SH, Chung MA, Quddus MR et al. The effect of neoadjuvant chemotherapy on estrogen and progesterone receptor expression and hormone receptor status in breast cancer. Am J Surg 2003; 186(4): 348-350.

19. Lower EE, Glass E, Blau R et al. HER-2/neu expression in primary and metastatic breast cancer. Breast Cancer Res Treat 2009; 113: 301-306.

20. Wilking U, Skoog L, Elmberger G et al. HER2 gene amplification (HER2) and hormone receptor expression (ER/PR) in early $(\mathrm{EBC})$ and metastatic breast cancer (MBC) in the same patients. J Clin Oncol 2007; 25 (18 Suppl): Abstract 1023.

21. Guarneri V, Giovannelli S, Ficarra G et al. Comparison of HER-2 and hormone receptor expression in primary breast cancers and asynchronous paired metastases: impact on patient management. Oncologist 2008; 13: 838-844.

22. Carreño G, Del Casar JM, Corte MD et al. Local recurrence after mastectomy for breast cancer: analysis of clinicopathological, biological and prognostic characteristics. Breast Cancer Res Treat 2007; 102: 61-73.

23. Moran MS, Haffy BG. Loco-regional breast cancer recurrence: prognostic groups based on patterns of failure. Breast J 2002; 8: 81-87.

24. Colleoni M, O'Neill A, Goldhirsch A et al. Identifying breast cancer patients at high risk for bone metastases. J Clin Oncol 2000; 18(23): 3925-3935.

25. Nemoto R, Uchida K, Tsutsumi M et al. A model of localized osteolysis induced by the MBT-2 tumor in mice and its responsiveness to etidronate disodium. $J$ Cancer Res Clin Oncol 1987; 113: 539-543.

26. Gnant M, Mlineritsch B, Schippinger $W$ et al. Endocrine therapy plus zoledronic acid in premenopausal breast cancer. N Engl J Med 2009 360(7): 679-691.

27. Waeber M, Castiglione-Gertsch M, Dietrich D et al. Adjuvant therapy after excision and radiation of isolated postmastectomy locoregional breast cancer recurrence: definitive results of a phase III randomized trial (SAKK 23/82) comparing tamoxifen with observation. Ann Oncol 2003; 14: 1215-1221. 